\title{
Understanding the Role of Mathematics in Engineering Problem Solving
}

\section{DeLean Tolbert, Purdue University, West Lafayette (College of Engineering)}

DeLean Tolbert is a Research Fellow in the Department of Industrial and Manufacturing Systems Engineering at the University of Michigan-Dearborn. Her work intersects both design research and research on the access and experiences of underrepresented communities' along engineering pathways. Currently, she is exploring how African American youth access capital to develop engineering skills and how those skills can be transferred to engineering education settings.

Dr. Monica E. Cardella, Purdue University, West Lafayette (College of Engineering)

Monica E. Cardella is the Director of the INSPIRE Institute for Pre-College Engineering Education and is an Associate Professor of Engineering Education at Purdue University. 


\section{Understanding the Role of Mathematics in Engineering Problem Solving}

\section{Abstract}

The core of engineering includes both mathematical thinking and design thinking. Previous work has focused on design thinking skills and behaviors exhibited by novices, experts, first-year engineering students, and senior engineering students. In this NSF-funded study, we sought to explore the interplay between mathematics and design by observing students from diverse disciplinary backgrounds engage in mathematical and design thinking as they solve a design task. Students in engineering majors $(n=17)$ and mathematics majors $(n=13)$ were recruited to work independently on a design task. In this paper, we present analysis methods, data, and findings from two seniors: one undergraduate engineering major and one undergraduate mathematics major. Ultimately the research team anticipates making comparisons between final design quality and using narrative analysis to explore design and mathematics processes. The findings from the complete data set will support deepened understanding of the role mathematics and design thinking in solving engineering design tasks and will create a baseline for comparing engineering student's mathematics and design behaviors in future analysis.

\section{Introduction}

Researchers have found that when engineering students have knowledge and a large repertoire of problem solving strategies they provide more complete and correct answers to the given problems ${ }^{1}$. Mathematics contributes to the core of engineering and serves as a source of knowledge from which engineering students can draw from. Thus, engineering students must have an ability to apply mathematical knowledge and skills to problem solving and engineering design tasks. Simply having mathematical or engineering knowledge without understanding of how to apply the learned strategies can limit a student's ability to provide correct answers ${ }^{2}$.

Aspects of mathematical thinking are characterized as analytical skills, in this study. Engineers utilize these analytical skills to develop and assess solutions for feasibility, efficiency, and correctness. Schoenfeld ${ }^{3}$ defines mathematical thinking as a culmination of an individual's "mathematical knowledge, their ability to apply mathematical problem solving strategies, the effective use of cognitive resources, having a mathematical perspective, and engaging in mathematical practices." See Appendix A for a list of observable mathematical aspects. Engineering curriculum is largely designed around a core mathematics curriculum, which typically begins with Calculus and concludes with Differential equations. Therefore, it is important to understand how engineers apply analytical thinking skills and other mathematical practices to engineering tasks.

In previous studies, researchers have explored the strategies that engineers use to solve design problems ${ }^{4}$. Research studies focused on understanding the behaviors of experts and novices have demonstrated that varied application of problem solving approaches or heuristics can lead to more correct and robust solutions ${ }^{5}$. Much of the previous work related to expert novice studies focused on the engineering students' and professionals' movement through design steps as they completed the given design task. This work included the think aloud protocol design, in which the research participant completes a design task, while at the same time verbally communicating 
his or her actions (i.e., talking through actions as they are completed). While this approach is limited in that some individuals tend to report past events, summarize activities, talk in muffled voice, or are not able to verbalize their quick-thinking processes. This approach is a very strong method to understand the process that a participant used to arrive at a complete solution ${ }^{6}$. When think aloud protocol study design is coupled with video data recordings and analysis, the trustworthiness is improved.

In mathematics research, it was found that students who articulated their solution processes are more "mathematically competent." In a study, which investigated Turkish undergraduate students, it was found that when students provided a complete description of their process then their solutions were correct or partially correct ${ }^{7}$. Whereas, when students were unable to detail their thinking processes their submitted solutions yielded no answer or incorrect answers ${ }^{8}$. Therefore, our research objective is to explore the diverse problem solving process of mathematics and engineering students by coupling a think aloud protocol design with video data analysis.

To date research studies have explored problem solving behaviors in mathematics students and engineering design behaviors in engineering students. Prior to this work, research investigating engineering students' mathematical skills focused on mathematical modeling ${ }^{9,10}$. Yet, the core of engineering includes both analytical skills (operationalized as mathematical thinking) and creativity (operationalized as design thinking). By understanding how individuals from differing disciplines utilize their specific skills we anticipate identifying approaches that educators can use to teach engineering students effective mathematical and design thinking practices.

\section{Analysis Methods: Verbal Protocol and Narrative Analysis}

In previous work, researchers applied in-depth verbal protocol analysis (VPA) and reviewed transcripts of the three-hour design session using a line-by-line qualitative coding approach. This process and its application is detailed in other publications ${ }^{11}$. So in this text we briefly describe our application of VPA. Traditionally applied VPA allows both deep insights into specific behaviors and is a broad approach in that researchers can explore multiple research questions with one data set; however, it is a very time consuming process. Atman and Bursic ${ }^{11}$ acknowledge the time intensive strategy and provided alternative less time consuming but still rigorous approaches to investigating the problem-solving processes of research participants in a study using a think aloud study design. One of their recommendations, which informed the analysis approach for this study, was to collect the complete verbal protocol but isolate specific behaviors, segments, and processes of interest.

To identify segments of interest, the research team engaged in a narrative analysis approach to investigate the research participants' design processes. The research team resolved that there was tremendous value in analyzing the audio and video data. This approach allows the team to hear the verbalized thinking processes while observing each research participants' actions. The participant worked independently to solve a design task and was given up to three hours to complete the task. During the session, the study facilitator sat in the room with the participant to address questions, facilitate the follow-up interview and write research memorandums at fiveminute intervals, which were essentially observations of the participant and their actions. 


\section{An Application of the Analysis Methods}

\section{A. The Design Task and Experiment}

To demonstrate the application of the narrative analysis approach we present the data from two seniors who respectively represent mathematics and engineering disciplines. The students were recruited as part of the larger study, which included first-year first and second semester undergraduate engineers, and seniors in mathematics, design and engineering disciplines. There are a few steps of application for the verbal protocol approach ${ }^{11}$. At the start of the design session, the research participant must talk aloud as they solve a short open-ended design problem and a mathematics equation. This allows them to gain familiarity with the talk aloud method. Once they have completed the two practice problems, the participant receives the one-page design task statement, which they must read aloud. After reading the statement, the participant has up to three hours to complete the task. The statement details constraints and encourages the participant to request information. The participant has access to a resource box with miscellaneous tools (i.e., a calculator, post-it notes, pencils, pens, colored pencils, rulers, etc.). They have additional access to the facilitator and information binder (the participant must ask for specific information) and an internet-connected computer. Refer to figure 1 for the design task statement.

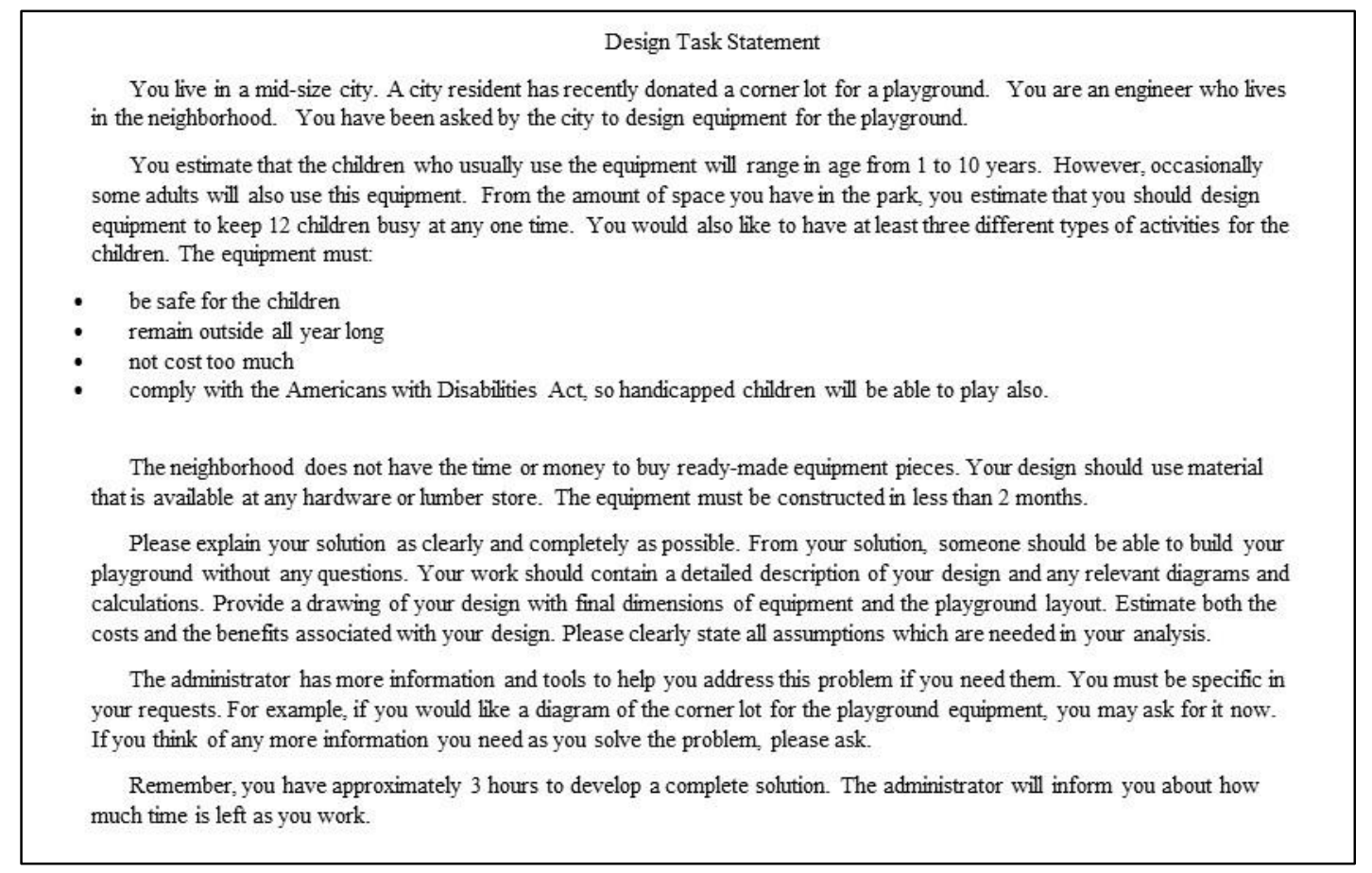

Figure 1: Study Design Task Statement

\section{B. Description of the Data}

Each design session lasts up to three hours. There is a scheduled ten-minute break and an approximately 25-minute follow-up interview. Each session is video and audio recorded, the onsite researcher records brief field observations at timed intervals, the web browsing history is saved, and the research participants submit their final design solution. 


\section{Data Preparation - Segmentation}

The video data were segmented into five-minute units. The segmentation was useful for this project because the unitized segments make the large data set much more manageable for analysis. It also allows researchers to identify smaller video experts of specific mathematical or engineering behaviors.

\section{Coding Manual and Research Framework}

Over the course of this project the research team developed and tested various coding frameworks for analyzing the data. Three components guided the design of the final coding manual and research framework. The resulting coding scheme is represented in figures 2 and 3. The first component is Cardella's adaptation of Schoenfeld's ${ }^{3,12}$ framework which includes five aspects of mathematical thinking that can be operationalized using the language presented in the table. For this study, we only focus on three observable aspects which were developed from research based findings related to how mathematics students and engineering students use mathematics problem solving approaches, namely: use of resources, problem solving strategies, and mathematical practices. The second component of the coding framework includes the design steps ('design process steps') which were developed from previous research studies which investigated the design process of expert and novice engineers ${ }^{4,5,13}$. The design steps include problem definition, information gathering, idea generation, modeling, feasibility analysis, evaluation, decision-making, and communication. Finally, we were interested in evaluating the quality of the final playground design solutions. The quality-scoring rubric ${ }^{5,13}$, was used to score each participants' final solution. The rubric measures quality in three parts: 1) criteria that all playgrounds are expected to meet $(n=40)$, supplementary criteria for included materials and items (i.e., metal, wood, chains, etc.), and qualitative ratings for creativity (e.g., diversity of activities, aesthetics, protection from injury, uniqueness, and technical feasibility). For each of the items the participant could be scored on a scale from one to five.

\begin{tabular}{|c|c|c|}
\hline & $\begin{array}{l}\text { Problem } \\
\text { Definition }\end{array}$ & Defining what the problem really is. \\
\hline & $\begin{array}{l}\text { Gather } \\
\text { Information }\end{array}$ & $\begin{array}{c}\text { Searching for and collecting information (i.e., facts, data) needed to solve the } \\
\text { problem }\end{array}$ \\
\hline & Generate Ideas & $\begin{array}{l}\text { Thinking up potential solutions (or parts of potential solutions) to the problem. } \\
\text { Integrates the idea generation, ideascreening and idea selection processes. }\end{array}$ \\
\hline & Modeling & $\begin{array}{l}\text { Detailing how to build the solution (or parts of the solution) to the problem. } \\
\text { Applies to initial solution concepts as well as to the final design. }\end{array}$ \\
\hline & Feasibility & $\begin{array}{l}\text { Assessing and passing judgment on a possible or planned solution to the } \\
\text { problem. Determineworkability, does it meet constraints, criteria, etc.? }\end{array}$ \\
\hline & Evaluation & $\begin{array}{l}\text { Comparing and contrasting two (or more) solutions to the problem on a particular } \\
\text { dimension (or set of dimensions) such as strength or cost. }\end{array}$ \\
\hline & Decision & $\begin{array}{l}\text { Selecting one idea or solution to the problem (or parts of the problem) from } \\
\text { among those considered. }\end{array}$ \\
\hline & Communication & $\begin{array}{l}\text { The participant's communicating elements of the design in writing (e.g., } \\
\text { sketches, diagrams, lists, and reports), or with oral reports to parties such as } \\
\text { contractors and the community. }\end{array}$ \\
\hline
\end{tabular}




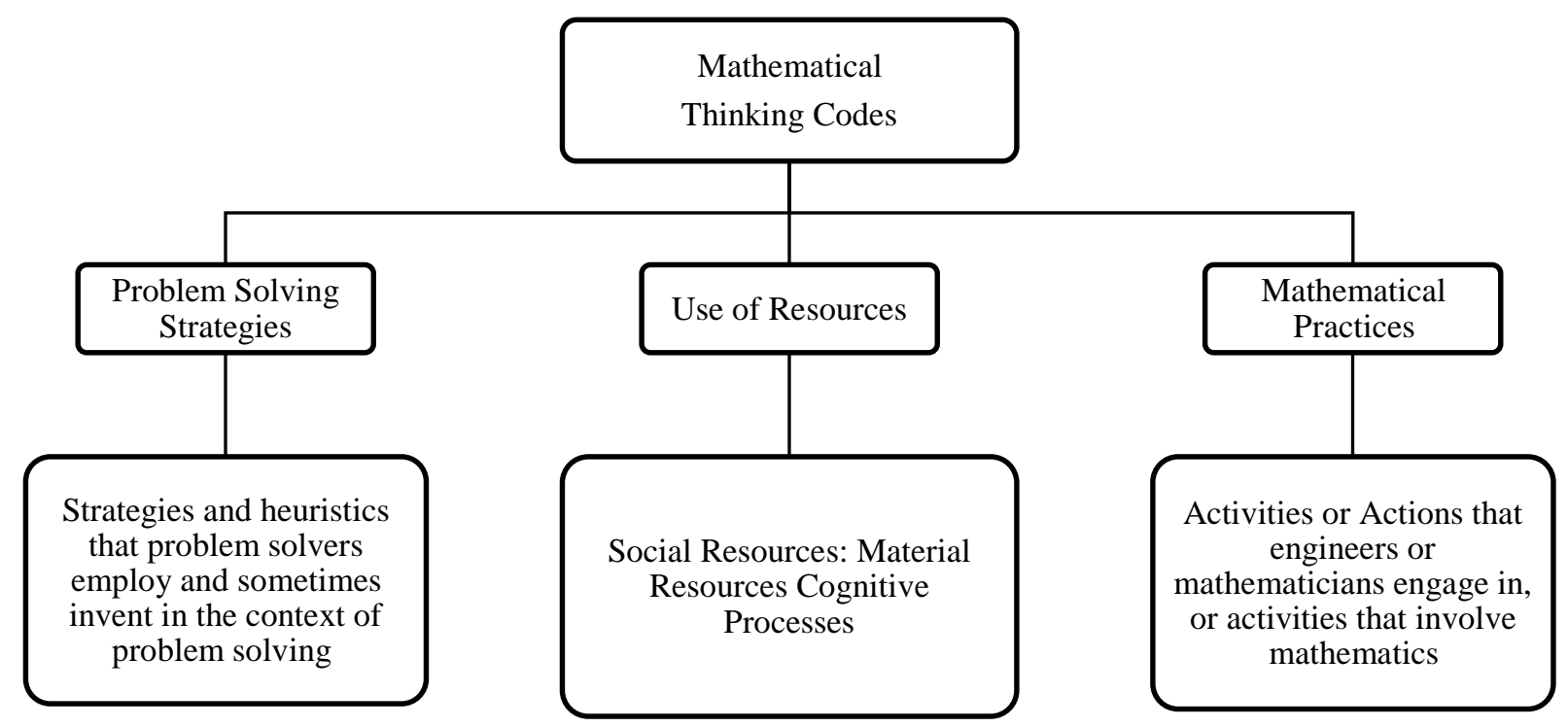

Figure 3 Mathematical Thinking Codes

\section{E. Summaries and Narratives}

Consistent with previously applied approaches, our objectives were to: 1) explore the participants' actions during each five-minute segment, 2) understand how they engage with different design steps, and 3) understand how they employ mathematical thinking strategies. Our final objective is to compare findings across the two participants ${ }^{7}$. We met these objectives using inductive coding and narrative analysis. The analysis methods, which we applied to the data of two research participants, illustrate the application of the VPA and narrative analysis approaches. Additionally, we will highlight the application of the coding manual and theoretical framework. Atman and Bursic ${ }^{11}$ used this approach to demonstrate the potential of applying verbal protocol analysis when exploring different design processes. The selection of these two participants may not represent the larger respective study population but will create a starting point for our continued analysis.

Rather than only coding the segments for the mathematical thinking and the design process codes, the research team wanted to explore the potential of using narrative analysis to investigate the problem solving process. Each narrative-coder on the team studied the manual and received training on how to make and record observations at five-minute intervals. The narrative-coder documented a narrative summary of each set three sequential 5-minute segments. The coder used the coding manual to support their observations, inferences, and interpretations. The coding manual helped to ground their observations in the framework and improved the trustworthiness ${ }^{14}$ of the study. Once the narrative summary was written for the entire video, a separate narrativecoder coded each of the segments and narratives using the coding manual as an inductive coding framework. 
Due to the length of each video recording ( three hours), we decided that after a team member was trained they would be responsible for a unique set of design session videos. Meaning that one narrative-coder coded each three-hour video. In the following sections, we present profiles of the selected participants, findings from the analysis, and implications for the application of our approach. With Schoenfeld-inspired plots ${ }^{15}$, we present summaries of the five-minute segments of data, and graphs and provide insights from the study methodology and the data.

\section{Descriptions of Sample Participants with Profiles}

In this section, we introduce the two senior participants whose data and findings demonstrate the application of the analysis method detailed above.

Casey, a female senior in engineering, spent most of her design session in the idea generation and information gathering design steps. Compared to many of the participants she developed an unorthodox idea generation and decision-making process. Her process included multiple phases. In phase one she documented potential solutions on individual but same colored post-it notes. In phase two, Casey eliminated infeasible design solutions. During phase three, she organized the post-it notes by equipment type. Finally, in phase four she used another colored post-it notes to document design details for each of the selected pieces of equipment. Her final submitted solution did not appear to be an organized structured document, rather her final submission was the culmination of her post-it notes and the details she noted for each piece of equipment. During minutes 45-60 of the study, the narrative-coder investigating Casey's work and process noted:

This segment starts out with her exhausting her researching online and reaching a point where all the ideas she has found so far have been listed. She then pulls out post-it notes, interestingly. She starts to go through her list and writes down her ideas. She color-codes her ideas, groups them by likeness, and discards some. Her groups seem to focus on big ideas. The extra notes are underneath with more details that might help her narrow down her search and make a design more solid. - Narrative Coder

Sieta, a senior mathematics senior, gathered a lot of information for the tasks and sketched often. She paid careful attention to the scale and created an exact scale for her playground layout. Drawing the exact scale is a behavior that was more common among the mathematicians but some engineering students also created a scale for their drawings. During minutes 30-45, the narrative coder investigating Sieta's process observed:

[Sieta] goes through each point on the safety regulations handout and thinks of an explanation for each danger and how to avoid it. [She] starts analyzing the dimensions of the equipment and [considers] how much space she has to utilize and then draws a general outline on paper. [She] maps out a scale to use for the playground. $1 \mathrm{~cm}=3 \mathrm{ft}$. - Narrativecoder 


\begin{tabular}{|c|c|c|c|c|c|c|}
\hline $\begin{array}{c}\text { Participant } \\
\text { pseudonym }\end{array}$ & $\begin{array}{c}\text { Discipline and } \\
\text { ranking }\end{array}$ & Sex & $\begin{array}{c}\text { Total } \\
\text { time on } \\
\text { task }\end{array}$ & $\begin{array}{c}\text { Design } \\
\text { experience }\end{array}$ & $\begin{array}{c}\text { Mathematics } \\
\text { experience }\end{array}$ & Final Score \\
\hline Casey & Senior Engineer & Female & 180 min & $\begin{array}{c}\text { Internship in } \\
\text { biomedical } \\
\text { device } \\
\text { design }\end{array}$ & $\begin{array}{c}\text { Vector Calculus, } \\
\text { Probability and } \\
\text { Statistics, Differential } \\
\text { Equations, Partial } \\
\text { Differential Equations }\end{array}$ & $36 / 72$ \\
\hline Sieta & $\begin{array}{c}\text { Senior } \\
\text { Mathematician }\end{array}$ & Female & 180 min & $\begin{array}{c}\text { No design } \\
\text { experience }\end{array}$ & $\begin{array}{c}\text { Calculus 1, 2, 3, and } \\
\text { Vector. Linear } \\
\text { Algebra 1 \& 2. } \\
\text { Differential } \\
\text { Equations, Logic, } \\
\text { Galois Theory, Real } \\
\text { Analysis and Abstract } \\
\text { Algebra Honors. }\end{array}$ & $41 / 74$ \\
\hline
\end{tabular}

Table 1 Participant information

V. Findings

A. Schoenfeld-inspired plots

Schoenfeld-inspired plots illustrate the moves between each students' design session segments and the coding framework. This approach was applied similarly in another recent study ${ }^{8}$. Schoenfeld ${ }^{15}$ originally used activity categories. However, we choose to identify movement between design steps and mathematical thinking aspects. Figures $4-5,8-9$ represent 40 minutes (i.e., segments 10-17 of 33) of Casey and Sieta's data and how they moved through the design steps and applied mathematical behaviors during the session. The figures display the design process and mathematics behaviors versus a time axis.

\section{B. Design steps}

In these 40 minute excerpts both Casey and Sieta engaged in seven of eight design steps, although throughout the complete design sessions, the participants engaged in all eight design steps. The excerpted segments in figures 4, 5, 8, and 9 take place in the last five minutes of the first hour and 40 minutes in to the second hour of the study. Casey is just beginning to use the post-it notes to organize her ideas that she continues to generate. She uses this process to categorize, discard and record details about selected solutions. She engages in very little modeling (as in detailing aspects of the design) but she remains open to new ideas that may challenge what she has already developed. She uses the computer to find information to help her make decisions about the equipment. 


\begin{tabular}{|c|c|c|c|c|c|c|c|c|}
\hline \multirow{2}{*}{ Time } & \multicolumn{8}{|c|}{ Design Steps (Casey) } \\
\hline & $\begin{array}{c}\text { Problem } \\
\text { Definition }\end{array}$ & Modeling & $\begin{array}{l}\text { Information } \\
\text { Gathering }\end{array}$ & $\begin{array}{c}\text { Idea } \\
\text { Generation }\end{array}$ & $\begin{array}{c}\text { Feasibility } \\
\text { Analysis }\end{array}$ & Evaluation & Decision & Communication \\
\hline 10 & & & & & & & & \\
\hline 11 & & & & & & & & \\
\hline 12 & & & & & & & & \\
\hline 13 & & & & & & & & \\
\hline 14 & & & & & & & & \\
\hline 15 & & & & & & & & \\
\hline 16 & & & & & & & & \\
\hline 17 & & & & & & & & \\
\hline
\end{tabular}

Figure 4: Schoenfeld-inspired plot for Casey’s Design Steps

During the same segments, Sieta focuses more on exact dimensions of playground equipment and the scale of the drawn layout. She also is considering potential equipment and uses the internet to search for information. However, she spends much more time documenting aspects of the design through modeling and communication. Sieta engages in fewer design steps per segment than Casey does.

\begin{tabular}{|c|c|c|c|c|c|c|c|c|}
\hline \multirow{2}{*}{ Time } & \multicolumn{8}{|c|}{ Design Steps (Sieta) } \\
\hline & $\begin{array}{l}\text { Problem } \\
\text { Definition }\end{array}$ & Modeling & $\begin{array}{c}\text { Information } \\
\text { Gathering }\end{array}$ & $\begin{array}{c}\text { Idea } \\
\text { Generation }\end{array}$ & $\begin{array}{c}\text { Feasibility } \\
\text { Analysis }\end{array}$ & Evaluation & Decision & Communication \\
\hline 10 & & & & & & & & \\
\hline 11 & & & & & & & & \\
\hline 12 & & & & & & & & \\
\hline 13 & & & & & & & & \\
\hline 14 & & & & & & & & \\
\hline 15 & & & & & & & & \\
\hline 16 & & & & & & & & \\
\hline 17 & & & & & & & & \\
\hline
\end{tabular}

Figure 5 Schoenfeld-inspired plot for Sieta's Design Steps

While the Schoenfeld-inspired plots represented excerpts from analysis, tree diagrams (see figures 6-7, 10-11) are used to represent how the participants engaged each design step throughout the entire design session. These diagrams were outputs from the Nvivo qualitative coding software. The tree diagrams are hierarchical representations of the aspects of the study framework (i.e., mathematical thinking and design steps) and their sub-codes. The figure is a visual representation of the patterns in the data with respect to the number of coded references (e.g., segments) for each code. 
As was initially noticed by the facilitator on site during data collection, most of Casey's time spent on design activities were coded as information gathering. Segments could be counted multiple times toward multiple design steps; therefore, for Casey there were 138 coded references. The total number of coded references are included in each of the nested boxes.

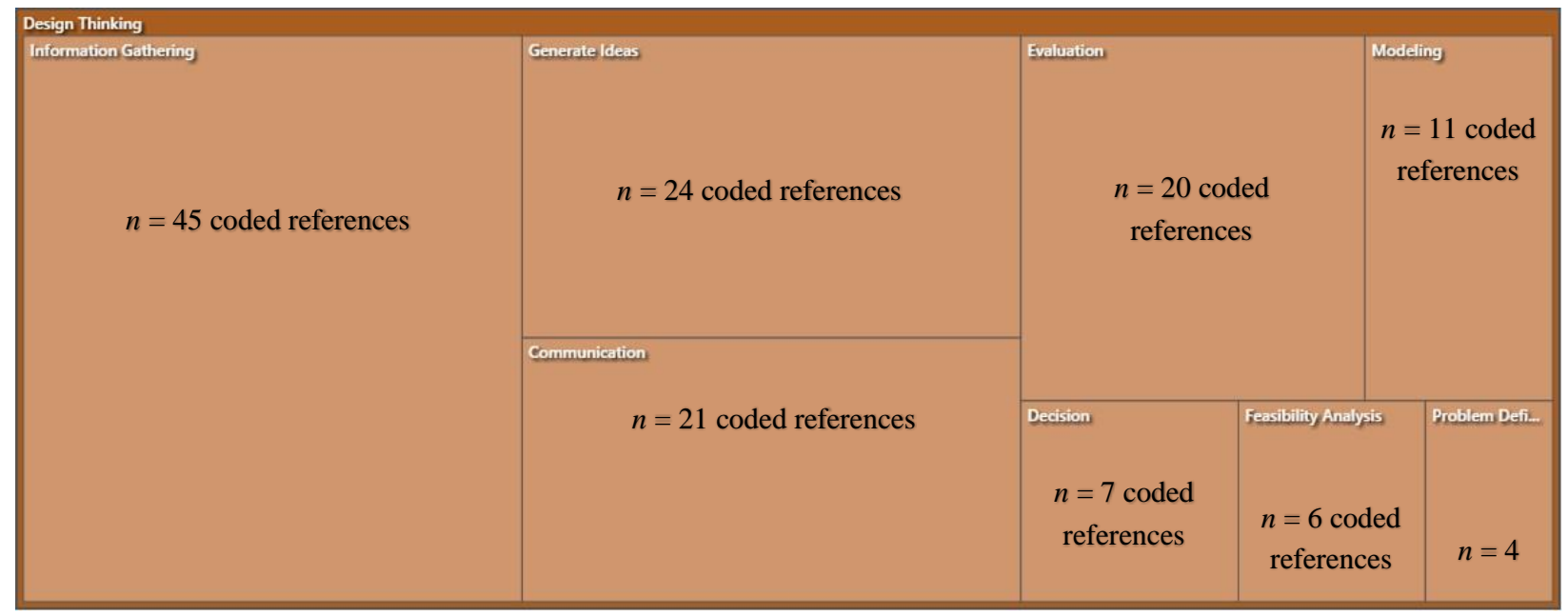

Figure 6 Casey's Tree Map for the Design Steps

As was observed during the design session, Sieta spent her time identifying types of the equipment and materials needed to build her design and communicating the final design aspects. See Figure 7 for instances of modeling, information gathering, and communication codes each had 19 coded references.

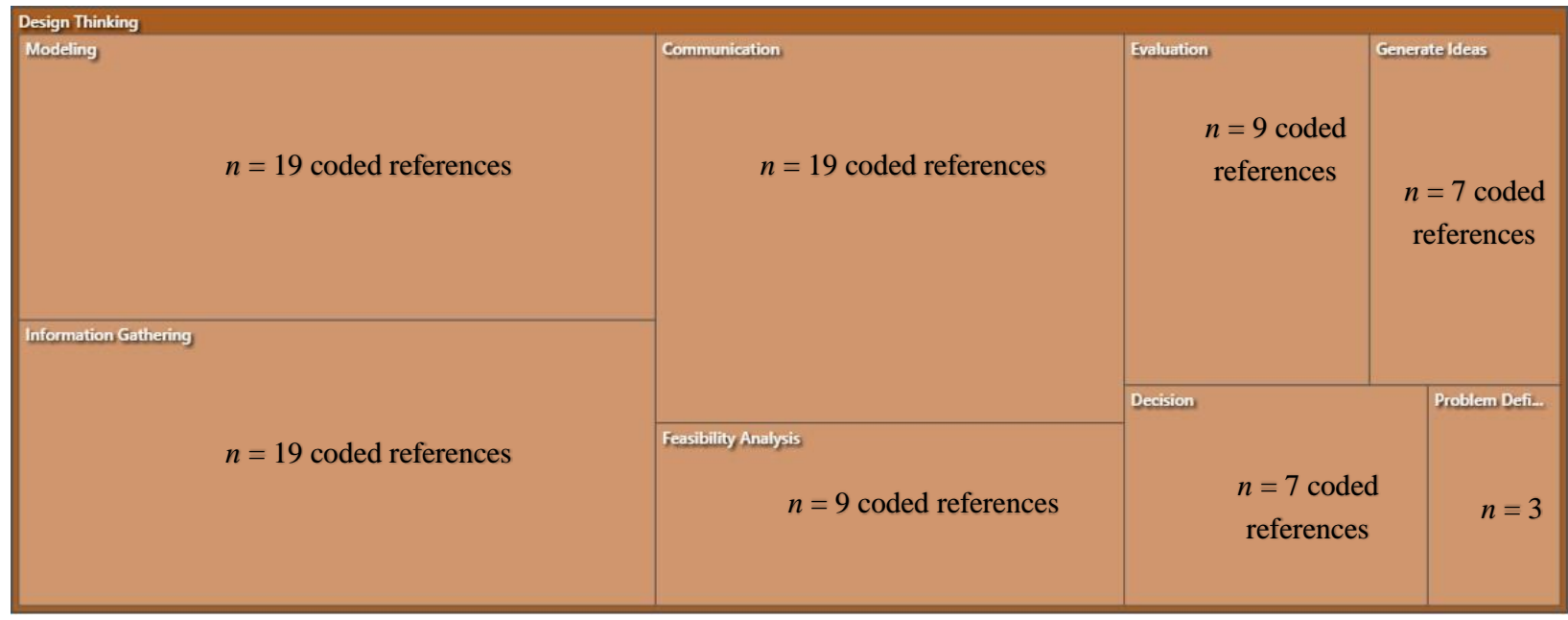

Figure 7 Sieta's Tree map for the Design steps

\section{Mathematical thinking}

The following Schoenfeld-inspired plots illustrate which mathematical thinking aspects each participant engaged in during segments 10-17. This same period examined in section above for design steps. The tree maps illustrate the subcategories of the mathematical thinking aspects and their frequency throughout the entire session. Refer to figures 8 and 9 for the tree maps. 
The plots as in figures 6 and 7 appear (at least for this excerpt) to reflect that Casey the engineer engages more in all aspects of the mathematical framework aspects. This provides evidence that, as an engineer, Casey applies some form of mathematical thinking in her design work. When we compare the specific sub-codes of each aspect, it was obvious that the participants use mathematical strategies and practices differently. They used similar resources for similar purposes. For example, using the ruler for drawing a scale, a calculator for crunching numbers, and the internet to search for costs and mathematical specifications.

\begin{tabular}{|c|c|c|c|}
\hline \multirow{2}{*}{ Time } & \multicolumn{3}{|c|}{ Mathematical Thinking Aspects (Casey) } \\
\hline & Use of Resources & Problem Solving Strategies & Mathematical Practices \\
\hline 10 & & & \\
\hline 11 & & & \\
\hline 12 & & & \\
\hline 13 & & & \\
\hline 14 & & & \\
\hline 15 & & & \\
\hline 16 & & & \\
\hline 17 & & & \\
\hline
\end{tabular}

Figure 8 Schoenfeld-inspired plot for Casey’s Mathematical Thinking Aspects

\begin{tabular}{|c|c|c|c|}
\hline \multirow{2}{*}{ Time } & \multicolumn{3}{|c|}{ Mathematical Thinking Aspects (Sieta) } \\
\hline & Use of Resources & Problem Solving Strategies & Mathematical Practices \\
\hline 10 & & & \\
\hline 11 & & & \\
\hline 12 & & & \\
\hline 13 & & & \\
\hline 14 & & & \\
\hline 15 & & & \\
\hline 16 & & & \\
\hline
\end{tabular}

Figure 9 Schoenfeld-inspired plot for Sieta's Mathematical Thinking Aspects

For the mathematical thinking codes, 79 references were coded for Casey and 91 references codes for Sieta. Casey engaged more with tools than did Sieta. Whereas, Sieta engaged more mathematical practices. In general, Sieta engaged more broadly in mathematical thinking activities. See figures 8 and 9 for the respective plots. 


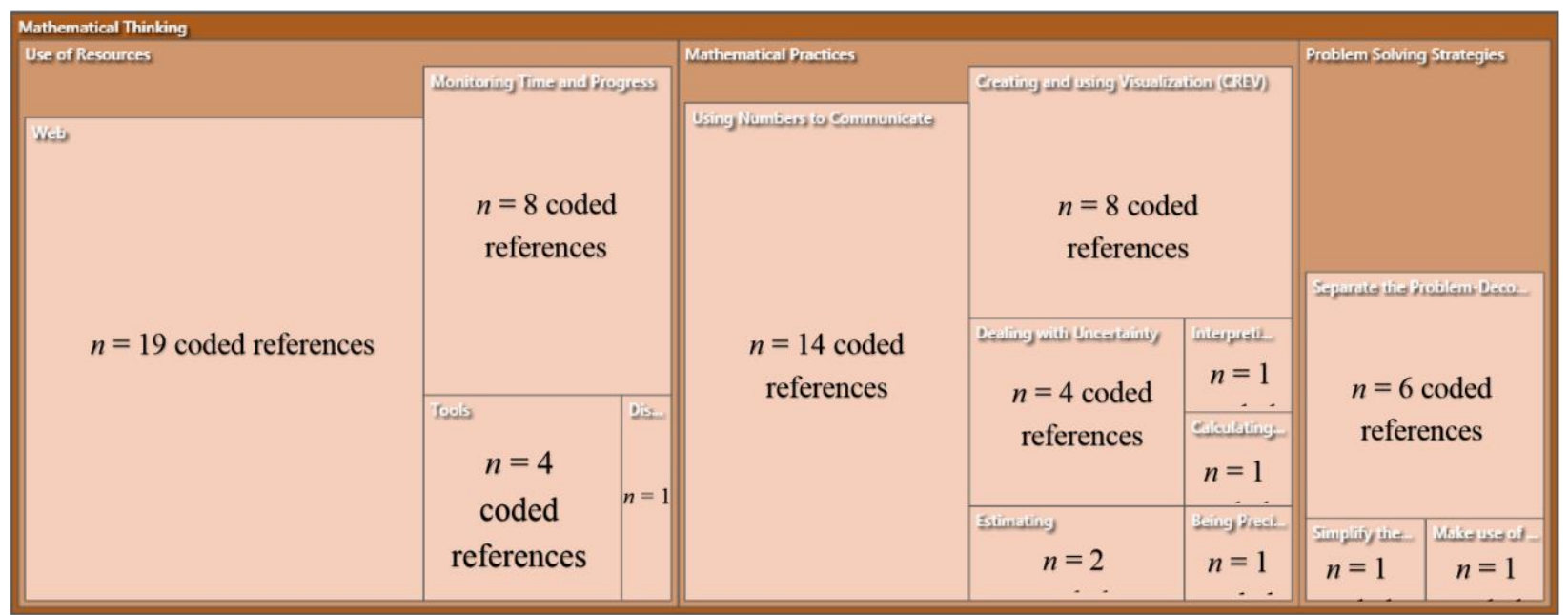

Figure 11 Casey's Tree Map for Mathematical Thinking

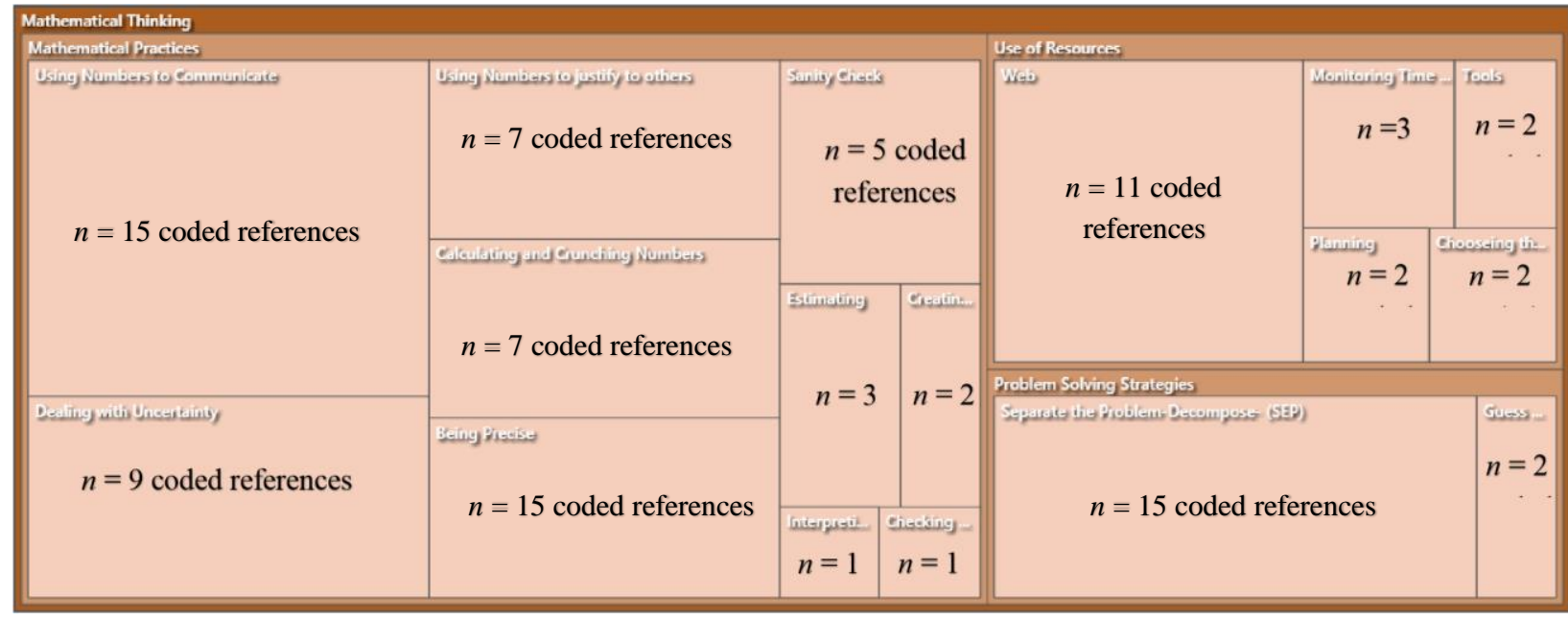

Figure 10 Sieta's Tree Map for Mathematical Thinking

\section{Analysis of all Participants}

As of submission, the research team is narrating and coding the remaining videos using the approach detailed above. We plan to compare processes used across the three senior groups, to make comparisons between the senior engineers and non-engineers, and to look for any patterns within the groups. Using the Schoenfeld-inspired plots, we can also begin to look for the interplay between mathematics and design thinking within each participant's design session. One way to visualize this interplay is to merge the separate mathematical thinking aspects and the design steps plots. See Appendix B for Casey and Sieta's merged plots. Finally, we anticipate that students who have a balanced approach to utilizing mathematical skills and design steps will have higher quality solutions. 


\section{Discussion}

The primary contribution of this work is the methodology used to analyze the data. Through the approach detailed in this manuscript, we observed and documented the various problems solving processes used by diverse disciplines and further detail a model for how engineering student can draw upon mathematics and design skills to develop high quality design solutions. The application of narrative analysis coupled with inductive coding helps to make design and mathematics behaviors more explicit and may inform teaching practice. This work may initiate innovative methods and provide content that transforms the ways the engineering students apply their design and mathematical skills as they solve engineering problems.

\section{Conclusions}

Narrative analysis can help engineering education researchers understand problem solving processes in ways that build upon previous verbal protocol analytical approaches. It allows us to look at the research participants' process in a less time consuming way than previous methods. Additionally, we anticipate that because we are not solely engaging in inductive coding, there is opportunity for us to observe new design process activities and behaviors.

\section{Acknowledgments}

We would like to acknowledge the efforts of Reis Lehman, Shrishti Jaganmohan, Ashley VanWormer, Sahil Bhalla, Zachary Beyer, Arman Shroff, Yicheng Zhou, Clayton Steele, Veronica Vera-Llano, and Nancy Garduno who have worked as undergraduate researchers on the project. We are grateful for each student who participated in this study. This material is based upon work supported by the National Science Foundation under Grant No. 1151019. Any opinions, findings, and conclusions or recommendations expressed in this material are those of the author(s) and do not necessarily reflect the views of the National Science Foundation. 


\section{References}

1. Erbas A, Okur S. Researching students' strategies, episodes, and metacognitions in mathematical problem solving. International Journal of Methodology. 2012;46(1):89102.

2. Osman SA, Mohd Salleh; Mohammad, Shahrin; Mokhtar, Mahani. Integrating Pertinent Elements of Critical Thinking and Mathematical Thinking Used by Practicing Civil Engineers in Grounded Theory Analysis. Journal for Social Sciences Research. 2015;8(3).

3. Schoenfeld A. Learning to think mathematically: Problem solving, metacognition, and sense-making in mathematics. In: Grouws D, ed. Handbook for Research on Mathematics Teaching and Learning New York: MacMillan; 1992:334-370.

4. Atman CJ, Chimka JR, Bursic KM, Nachtmann HL. A comparison of freshman and senior engineering design processes. Design Studies. 1999;20(2):131-152.

5. Atman CJ, Adams RS, Cardella ME, Turns J, Mosborg S, Saleem J. Engineering Design Processes: A Comparison of Students and Expert Practitioners. Journal of Engineering Education. 2007;96(4):359-379.

6. $\quad$ Ericsson KA. Protocol analysis: Verbal reports as data. Rev. ed.. ed. Cambridge, Mass.: MIT Press; 1992.

7. Gustafsson P, Jonsson G, Enghag M. The Problem- Solving Process in Physics as Observed When Engineering Students at University Level Work in Groups. European Journal of Engineering Education. 2015;40(4):380-399.

8. Gustafsson P, Gunnar J, Enghag M. The problem-solving process in physics as observed when engineering students at university level works in groups. European Journal of Engineering Education. 2015;40(4):380-399.

9. Cardella M, Tolbert D. "Problem Solving" in Engineering Research on Students' Engineering Design Practices and Mathematical Modeling Practices. Frontiers in Education Conference; 2014; Madrid, Spain.

10. Diefes-Dux HA, Zawojewski JS, Hjalmarson MA, Cardella M. A framework for analyzing feedback in a formative assessment system for mathematical modeling problems. Journal of Engineering Education. 2012;101(2).

11. Atman CJ, Bursic KM. Verbal protocol analysis as a method to document engineering student design processes. Journal of Engineering Education. 1998;87(2):121-132.

12. Schoenfeld AH. Mathematical thinking and problem solving. Hillsdale, N.J.: Hillsdale, N.J. : L. Erlbaum Associates; 1994.

13. Atman C, J.; Cardella, Monica, E.; Turns, Jennifer; Adams, Robin;. Comparing Freshman and Senior Engineering Design Processes: An In-depth Follow-up Study. Design Studies. 2005;26:325-357.

14. Miles MB, Huberman AM. Qualitative data analysis: An expanded sourcebook. 2nd ed. Thousand Oaks, California: SAGE Publications; 1994.

15. Schoenfeld AH. Mathematical problem solving. Orlando, FL: Academic; 1985. 
Appendix A

Mathematical Thinking Aspects

Problem solving strategies include:

- Start from Fundamentals

- Guess \& Verify

- Transform the Problem

- Simplify the Problem

- Separate the Problem into Smaller Problems

- Make Use of What You Have

Use of resources include:

- Tools (e.g. Excel, Simulation)

- Textbooks

- Monitoring Time and Progress

- Planning

- Choosing the Right Approach

- Discerning What's Relevant

- Website

Mathematical practices include:

- Having a Mathematical Perspective

- Using a Mathematical Vocabulary

- Using Numbers to Communicate

- Creating and Using Visualizations

- Using Numbers to Justify

- Mathematizing

- Dealing with Uncertainty

- Estimating

- Interpreting Numbers
- Being Precise

- Creating and Using Mathematical Models

- Calculating and Crunching Numbers

- Checking if Results Seem Reasonable

- Sanity Check 


\section{Appendix B}

Merged Schoenfeld-inspired plots to illustrate the interplay between mathematical and design thinking.

\begin{tabular}{|c|c|c|c|c|c|c|c|c|c|c|c|}
\hline \multirow{2}{*}{ Time } & \multicolumn{3}{|c|}{$\begin{array}{c}\text { Mathematical Thinking } \\
\text { Aspects }\end{array}$} & \multicolumn{8}{|c|}{ Design Steps (Casey) } \\
\hline & $\begin{array}{c}\text { Uge of } \\
\text { Resources }\end{array}$ & $\begin{array}{l}\text { Problem } \\
\text { Solving } \\
\text { Strategies }\end{array}$ & $\begin{array}{l}\text { Mathematical } \\
\text { Practices }\end{array}$ & \begin{tabular}{c|} 
Problem \\
Definition
\end{tabular} & Modeling & $\begin{array}{l}\text { Information } \\
\text { Gathering }\end{array}$ & $\begin{array}{c}\text { Idea } \\
\text { Generation }\end{array}$ & $\begin{array}{c}\text { Feasibility } \\
\text { Analysis }\end{array}$ & Evaluation & Decision & Communication \\
\hline 10 & & & & & & & & & & & \\
\hline 11 & & & & & & & & & & & \\
\hline 12 & & & & & & & & & & & \\
\hline 13 & & & & & & & & & & & \\
\hline 14 & & & & & & & & & & & \\
\hline 15 & & & & & & & & & & & \\
\hline 16 & & & & & & & & & & & \\
\hline 17 & & & & & & & & & & & \\
\hline
\end{tabular}

\begin{tabular}{|c|c|c|c|c|c|c|c|c|c|c|c|}
\hline \multirow{2}{*}{ Time } & \multicolumn{3}{|c|}{$\begin{array}{c}\text { Mathematical Thinking } \\
\text { Aspects }\end{array}$} & \multicolumn{8}{|c|}{ Design Steps (Sieta) } \\
\hline & $\begin{array}{c}\text { Uge of } \\
\text { Resources }\end{array}$ & $\begin{array}{c}\text { Problem } \\
\text { Solving } \\
\text { Strategies }\end{array}$ & $\begin{array}{c}\text { Mathematical } \\
\text { Practices }\end{array}$ & $\begin{array}{c}\text { Problem } \\
\text { Definition }\end{array}$ & Modeling & $\begin{array}{l}\text { Information } \\
\text { Gathering }\end{array}$ & $\begin{array}{c}\text { Idea } \\
\text { Generation }\end{array}$ & $\begin{array}{l}\text { Feasibility } \\
\text { Analysis }\end{array}$ & Evaluation & Decision & Communication \\
\hline 10 & & & & & & & & & & & \\
\hline 11 & & & & & & & & & & & \\
\hline 12 & & & & & & & & & & & \\
\hline 13 & & & & & & & & & & & \\
\hline 14 & & & & & & & & & & & \\
\hline 15 & & & & & & & & & & & \\
\hline 16 & & & & & & & & & & & \\
\hline 17 & & & & & & & & & & & \\
\hline
\end{tabular}

